\title{
Eigenfrequency Computation of Beam/Plate Carrying Concentrated Mass/Spring
}

\author{
Yin Zhang \\ State Key Laboratory of Nonlinear Mechanics \\ (LNM), \\ Institute of Mechanics, \\ Chinese Academy of Sciences, \\ Beijing 100190, People's Republic of China \\ e-mail: zhangyin@|nm.imech.ac.cn
}

With the adsorption of analyte on the resonator mass sensor, the system eigenfrequencies will shift due to the changes of inertial mass and structural rigidity. How to model those changes and formulate the eigenfrequency computation is very important to the mass sensor application, which results in different accuracies and requires different amounts of computation. Different methods on the eigenfrequency computation of a beam and a plate carrying arbitrary number of concentrated mass/spring are presented and compared. The advantages and disadvantages of these methods are analyzed and discussed. A new method called finite mode transform method (FMTM) is shown to have good convergence and require much less computation for a beam carrying concentrated mass/spring. Because the previous finite sine transform method (FSTM) has only been applied to compute the eigenfrequency of the plate with four edges simply supported carrying a single concentrated mass, here a generalized FSTM is also presented for the case of the same plate carrying arbitrary number of concentrated mass and spring. When the total number of concentrated mass and spring is small, FMTM and FSTM are demonstrated to be very efficient. [DOI: 10.1115/1.4002121]

Keywords: eigenfrequency, beam, plate, concentrated mass/spring, vibration, resonator

\section{Introduction}

The mass accretion on a structure results in the changes of the system resonant frequencies, traveling wave propagation, and damping [1-4]. These property changes are of pragmatic interest in many engineering applications and intrigue many investigations. These property changes are also utilized to develop various sensors. For example, resonator mass sensor operates by providing a frequency shift that is directly proportional to the inertial mass accreted upon it [5-17]. The resonator mass sensor is attractive in part because of its high sensitivity and frequency stability, making it possible and suitable to detect tiny mass changes. The capability of nanomechanical resonator to detect a single $E$. Coli bacteria [5] and virus [6] has been demonstrated. With the advances of micro-/nanoelectromechanical system (MEMS/NEMS) technologies, the mass detecting sensitivity now has been pushed to the scale of zeptogram $\left(10^{-21} \mathrm{~g}\right)[7]$.

The relation between the structure eigenfrequency shift and the accreted mass is the most important thing in the resonator mass sensor modeling. In this paper, a continuum mechanics approach is used. Although the resonator mass sensor with nanometer scale has been fabricated, recent molecular dynamics simulation and initial experiments appear to indicate that the continuum mechanics model is still valid for the structures with the cross section of the order of tens of lattice constants [8]. The resonator mass sensors in general are the plate [1-4] or beam [5-17] type of structure. Compared with the whole sensor structure, the size of accreted mass is often very small $[5,6,9-11]$. Therefore, the accreted mass can be modeled as a concentrated mass. One degree of freedom (DOF) model is the simplest one to describe the relation between the eigenfrequency shift and accreted mass $[6,10-13,16,17]$. 1DOF model uses the spring-mass system, which requires the effective spring stiffness and mass to be known a priori for the computation. To model the resonator mass sensor of

Contributed by the Technical Committee on Vibration and Sound of ASME for publication in the Journal OF VIBRATION AND Acoustics. Manuscript received October 19, 2009; final manuscript received May 26, 2010; published online March 3, 2011. Assoc. Editor: Wei-Hsin Liao. continuum system as 1DOF spring-mass system, the first step is often to use statics to find the effective spring stiffness of a continuum system without the presence of concentrated mass/spring $[10,16,17]$. An alternative way of determining the effective spring stiffness is to use the Galerkin method with one mode approximation [18]. The second step is to find the effective mass by comparing the resonant frequency obtained by $1 \mathrm{DOF}$ model and continuum theory. The effective spring stiffness found by statics (without a special treatment) is only valid for system vibrating around or lower than the fundamental eigenfrequency (the eigenfrequency of the first mode) because only the first mode shape is similar to the static deflection. Recent experiments $[11,12]$ demonstrate that much higher mass sensitivity can be achieved when the resonator mass sensor is driven at/around higher eigenfrequencies. The effective spring stiffness of the continuum system driven at/around higher eigenfrequencies can still be found via statics by adding constraints as demonstrated by Tseytlin [16], which is quite complex and difficult. In 1DOF model, the effective spring stiffness is often assumed unchanged and the inertial mass change is thus the only mechanism responsible for the eigenfrequency shift $[6,10-13,16,17]$. However, the recent experiment by Ramos et al. [5] shows a surprising $24 \%$ eigenfrequency increase of the mass sensor system with the adsorption of an E. Coli bacteria. Because the inertial mass increase can only result in the decrease of the system eigenfrequencies, Ramos et al. [5] argued that the adsorption of an E. Coli bacteria must also increase the system rigidity. In this paper, the concentrated spring is also incorporated in the model to account for such possible rigidity increase. With this possible rigidity increase effect, using statics to determine the effective spring stiffness for higher modes becomes much more complex and difficult. In addition, because the effective spring stiffness is found via statics, the shape of static deflection curve is thus implicitly assumed the same as that of dynamic one. With large concentrated mass or spring stiffness, which makes the dynamic deflection curve significantly different from the static one, 1DOF model may not be accurate. Similarly, the Rayleigh method uses the static deflection curve to approximate the system dynamic deflection curve $[19,20]$. Instead of using spring-mass system, the governing equation of the Rayleigh method is obtained 
by equating the maximum kinetic energy and maximum potential energy of the system $[19,20]$. The effective spring stiffness and effective mass can also be found by the Rayleigh method $[19,20]$. The accuracy of the Rayleigh method depends on how closely one can approximate the dynamic deflection curve [20]. For example, the approximation curve used by Timoshenko and Young [19], which is derived from statics, is shown to be applicable only in certain scenarios [20]. It is difficult and often experience dependent to find such close approximation as reflected in Low's complex construction scheme of the approximation curves for the Rayleigh method [20]. The Rayleigh method, as commented by Chen [21], is not easily applicable to the general case of the eigenfrequency computation of a beam carrying concentrated mass.

In the analytical method [9,22-26], the influence of concentrated mass/spring on the system eigenfrequencies is embodied in the so-called compatibility/equilibrium conditions [9,22], or continuity conditions [23], or boundary conditions [24,25], or transition conditions [26] at the location of concentrated mass/spring. In the analytical method, concentrated mass/spring does not appear in the governing equation and no approximation for structure dynamic deflection is required. The eigenvalue problem formulation by the analytical method results in a transcendental equation, which requires numerical method for the solution [9,22-26]. As analyzed later in this paper, the computation effort of the analytical method can be tremendous. Both the Galerkin method and finite mode transform method (FMTM) use the Dirac delta function to incorporate the influence of concentrated mass/spring into the governing equations $[18,27,28]$ and use the mode shapes of a uniform beam to approximate the dynamic deflection curve of the beam carrying concentrated mass/spring. Here an analytical expression for the system eigenfrequency is derived from the Galerkin method by using one mode approximation, which is suitable for resonator to detect small mass or spring stiffness change. FMTM developed in this paper is similar to Amber-Rao's finite sine transform method (FSTM) used in the rectangular plate with four edges simply supported carrying a single concentrated mass [29]. The name of finite sine transform method is given by $\mathrm{Wu}$ and Luo [30] because the dynamic deflection of the plate with four edges simply supported is approximated by the double sine functions and an integral transform is used [29]. Unlike FSTM, which can only be applied to the plate with four edges simply supported, FMTM is capable of computing the eigenfrequencies of the beam with different (arbitrary) boundary conditions. Compared with the analytical method, FMTM also requires less computation.

In the eigenfrequency computation of a plate carrying concentrated mass/spring, various methods are developed, such as FSTM [29], analytical-numerical-combined method (ANCM), and finite element method (FEM) [30], Rayleigh method [31], Laplace transform [32], and Green's function [33]. Because concentrated mass/spring can severely distort the plate dynamic deflection curve, large number of series/elements are needed for good approximation, which results in large computation. For example, Chiba and Sugimoto's eigenfrequency computation is an eigenvalue problem of a $50 \times 50$ matrix [31]; Wu and Luo's is an eigenvalue problem of a $175 \times 175$ matrix $($ FEM) and a $30 \times 30$ matrix (ANCM) [30], respectively. FSTM, as pointed by $\mathrm{Wu}$ and Luo [30], has only been applied to the simple case of the plate carrying single concentrated mass. In this paper, a generalized FSTM for a plate carrying arbitrary number of concentrated mass and spring is presented. Although FSTM also uses many modes to approximate the dynamic deflection, it is demonstrated that the eigenfrequency computation formulated by FSTM in essence is an eigenvalue problem of an $(r+s) \times(r+s)$ matrix, where $r+s$ is the total number of concentrated mass and spring. When $r+s$ is small, FSTM is very efficient because much less computation is required.

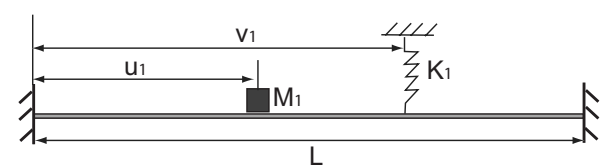

Fig. 1 The schematic diagram of a beam carrying a concentrated mass and spring. The concentrated mass is located at $x=u_{1}$ and the concentrated spring is at $x=v_{1}$.

\section{Eigenfrequency Computation of a Beam Carrying Concentrated Mass and Spring}

2.1 Analytical Method. The detailed formulation by the analytical method on the eigenvalue problem of a uniform beam carrying a single concentrated mass is presented by Srinath and Das [23] and that of a uniform beam carrying a single concentrated spring is given by Plaut et al. [26]. Here the formulation by the analytical method of an eigenfrequency problem on the beam carrying a concentrated mass and a concentrated spring is illustrated in detail. The general formulation for the beam carrying arbitrary number of concentrated mass and spring is also discussed.

Figure 1 shows the schematic diagram of a beam carrying a concentrated mass $M_{1}$ at $x=u_{1}$ and a concentrated translational spring $K_{1}$ at $x=v_{1}$. The governing equation of a vibrating beam is

$$
m \frac{\partial^{2} w}{\partial t^{2}}+E I \frac{\partial^{4} w}{\partial x^{4}}=0
$$

where $m$ is the beam mass per unit length, $E$ is Young's modulus, $I$ is the moment of inertia, and $I=b h^{3} / 12$ for a rectangular cross section beam ( $b$ and $h$ are the beam width and thickness, respectively). Although Eq. (1) is the exactly same governing equation of a uniform beam, it needs to emphasize that $w$ here is the beam dynamic transverse displacement with the presence of concentrated mass/spring. In the analytical method, the effects of concentrated mass/spring are embodied in the so-called compatibility/ continuity/transition conditions [9,22-26], not in the governing equation. In order to compare our results with the dimensionless results presented by Low [22], the following dimensionless quantities are introduced:

$$
\begin{gathered}
W=\frac{w}{L}, \quad \xi=\frac{x}{L}, \quad \mu_{e}=\frac{u_{e}}{L}, \quad \nu_{e}=\frac{v_{e}}{L}, \quad \tau=\sqrt{\frac{E I}{m L^{4}}} t \\
\alpha_{e}=\frac{M_{e}}{m L}, \quad \beta_{e}=\frac{K_{e} L^{3}}{E I}
\end{gathered}
$$

The subscript $e$ of $\mu$ starts from 1 to $r$ ( $r$ is the total number of concentrated masses) and the subscript $e$ of $\nu$ starts from 1 to $s$ ( $s$ is the total number of concentrated springs). Equation (1) is now nondimensionalized as follows:

$$
\frac{\partial^{2} W}{\partial \tau^{2}}+\frac{\partial^{4} W}{\partial \xi^{4}}=0
$$

$W(\xi, \tau)$ is assumed to have the following solution form:

$$
W(\xi, \tau)=Y(\xi) e^{i \omega \tau}= \begin{cases}Y_{1}(\xi) e^{i \omega \tau}, & 0 \leq \xi \leq \mu_{1} \\ Y_{2}(\xi) e^{i \omega \tau}, & \mu_{1}<\xi \leq \nu_{1} \\ Y_{3}(\xi) e^{i \omega \tau}, & \nu_{1}<\xi \leq 1\end{cases}
$$

In the above equation $\mu_{1}<\nu_{1}$ is assumed. $\omega$ here is the eigenfrequency of the system with the presence of concentrated mass and spring. Substituting Eq. (4) into Eq. (3), $Y_{1}, Y_{2}$, and $Y_{3}$ are solved as follows:

$$
\begin{aligned}
& Y_{1}(\xi)=C_{1} \sin (\Lambda \xi)+C_{2} \cos (\Lambda \xi)+C_{3} \sinh (\Lambda \xi)+C_{4} \cosh (\Lambda \xi) \\
& Y_{2}(\xi)=C_{5} \sin (\Lambda \xi)+C_{6} \cos (\Lambda \xi)+C_{7} \sinh (\Lambda \xi)+C_{8} \cosh (\Lambda \xi)
\end{aligned}
$$


$Y_{3}(\xi)=C_{9} \sin (\Lambda \xi)+C_{10} \cos (\Lambda \xi)+C_{11} \sinh (\Lambda \xi)+C_{12} \cosh (\Lambda \xi)$

$\Lambda$ is defined as $\Lambda=\sqrt{\omega}$. There are 12 unknown constants $C_{i}$ and therefore, 12 boundary and matching conditions in total are needed for the eigenvalue problem formulation. The four matching conditions at $\xi=\mu_{1}$ of a concentrated mass are [22,23]

$$
\begin{gathered}
Y_{1}\left(\mu_{1}\right)=Y_{2}\left(\mu_{1}\right), \quad \frac{d Y_{1}}{d \xi}\left(\mu_{1}\right)=\frac{d Y_{2}}{d \xi}\left(\mu_{1}\right) \\
\frac{d^{2} Y_{1}}{d \xi^{2}}\left(\mu_{1}\right)=\frac{d^{2} Y_{2}}{d \xi^{2}}\left(\mu_{1}\right), \quad-\frac{d^{3} Y_{1}}{d \xi^{3}}\left(\mu_{1}\right)+\frac{d^{3} Y_{2}}{d \xi^{3}}\left(\mu_{1}\right)=\alpha_{1} \omega^{2} Y_{1}\left(\mu_{1}\right)
\end{gathered}
$$

The first three matching conditions indicate the continuity of the displacement, slope, and curvature, respectively. The fourth one is derived from the dynamic equilibrium [23]. The above four matching conditions do not incorporate the effect of the concentrated mass rotary inertia [23]. The other four matching conditions at $\xi=\nu_{1}$ of a concentrated spring are [26]

$$
\begin{gathered}
Y_{2}\left(\nu_{1}\right)=Y_{3}\left(\nu_{1}\right), \quad \frac{d Y_{2}}{d \xi}\left(\nu_{1}\right)=\frac{d Y_{3}}{d \xi}\left(\nu_{1}\right) \\
\frac{d^{2} Y_{2}}{d \xi^{2}}\left(\nu_{1}\right)=\frac{d^{2} Y_{3}}{d \xi^{2}}\left(\nu_{1}\right), \quad \frac{d^{3} Y_{2}}{d \xi^{3}}\left(\nu_{1}\right)-\frac{d^{3} Y_{3}}{d \xi^{3}}\left(\nu_{1}\right)=\beta_{1} Y_{2}\left(\nu_{1}\right)
\end{gathered}
$$

The following four boundary conditions hold for a clampedclamped beam, which indicate the vanishing of the displacement and slope at the ends:

$$
Y_{1}(0)=0, \quad \frac{d Y_{1}}{d \xi}(0)=0, \quad Y_{3}(1)=0, \quad \frac{d Y_{3}}{d \xi}(1)=0
$$

Equations (6)-(8) give 12 matching and boundary conditions in total. Therefore, with the substitution of Eq. (5) into the above 12 equations, the eigenfrequency $\omega$ can be found. In general, when a new concentrated mass/spring is added, four new matching conditions at the location will be generated. For the case of total $r$ $+s$ concentrated masses and springs, $Y(x)$ is divided into $r+s+1$ subdomains. There are total $4(r+s+1)$ unknown constants of $C_{i} \mathrm{~s}$. The eigenfrequencies are found by setting the determinant of the $4(r+s+1) \times 4(r+s+1)$ matrix to be zero.

Here the eigenvalue problem formulation of a clampedclamped beam with a concentrated mass $\left(M_{1}\right)$ at the center in Ref. [9] is presented for comparison. Instead of explicitly dividing the beam span into two subdomains, Ilic et al. [9] formulated the problem by applying the symmetry and only one solution of a subdomain is used. The solution form of $Y_{1}(\xi)$ still holds as $Y_{1}(\xi)=C_{1} \sin (\Lambda \xi)+C_{2} \cos (\Lambda \xi)+C_{3} \sinh (\Lambda \xi)+C_{4} \cosh (\Lambda \xi)$ (now in the problem formulated by Ilic et al. [9], $Y_{1}(\xi)$ is the solution for the $0 \leq \xi \leq 1 / 2$ subdomain). At $\xi=0$, the clamped boundary conditions require the vanishing of both deflection and slope, which results in the following two equations:

$$
C_{2}+C_{4}=0, \quad C_{1}+C_{3}=0
$$

In conjunction with Eq. (9), $Y_{1}$ can now be written as $Y_{1}(\xi)$ $=C_{1}[\sin (\Lambda \xi)-\sinh (\Lambda \xi)]+C_{2}[\cos (\Lambda \xi)-\cosh (\Lambda \xi)]$. At $\xi=1 / 2$, the slope vanishes because of the symmetry; therefore,

$$
C_{1}\left(\cos \frac{\Lambda}{2}-\cosh \frac{\Lambda}{2}\right)-C_{2}\left(\sin \frac{\Lambda}{2}+\sinh \frac{\Lambda}{2}\right)=0
$$

The fourth one is derived from $-d^{3} Y_{1} / d \xi^{3}=\alpha_{1} \omega^{2} / 2 Y_{1}$, which corresponds to the fourth matching condition in Eq. (6). Because of the symmetry, here a factor of $1 / 2$ is added. Now the fourth condition is rewritten as follows:

$$
\begin{gathered}
C_{1}\left[\cos \frac{\Lambda}{2}+\cosh \frac{\Lambda}{2}-\frac{\alpha_{1} \Lambda}{2}\left(\sin \frac{\Lambda}{2}-\sinh \frac{\Lambda}{2}\right)\right]-C_{2}\left[\sin \frac{\Lambda}{2}-\sinh \frac{\Lambda}{2}\right. \\
\left.+\frac{\alpha_{1} \Lambda}{2}\left(\cos \frac{\Lambda}{2}-\cosh \frac{\Lambda}{2}\right)\right]=0
\end{gathered}
$$

The relation of $\Lambda^{2}=\omega$ is used during the derivation of the above equation. Equations (10) and (11) form a $2 \times 2$ matrix. To have nontrivial solution of $C_{1}$ and $C_{2}$, the following transcendental equation must be satisfied, which is obtained by setting the 2 $\times 2$ matrix determinant zero:

$$
\begin{gathered}
\left(\sin \frac{\Lambda}{2}+\sinh \frac{\Lambda}{2}\right)\left[\cos \frac{\Lambda}{2}+\cosh \frac{\Lambda}{2}-\frac{\alpha_{1} \Lambda}{2}\left(\sin \frac{\Lambda}{2}-\sinh \frac{\Lambda}{2}\right)\right] \\
-\left(\cos \frac{\Lambda}{2}-\cosh \frac{\Lambda}{2}\right)\left[\sin \frac{\Lambda}{2}-\sinh \frac{\Lambda}{2}+\frac{\alpha_{1} \Lambda}{2}\left(\cos \frac{\Lambda}{2}\right.\right. \\
\left.\left.-\cosh \frac{\Lambda}{2}\right)\right]=0
\end{gathered}
$$

Equation (12) is the characteristic equation given in Ref. [9] to determine the system eigenfrequency. Clearly, the above formulation by Ilic et al. fully utilizes the symmetry properties. However, it is not a general method to formulate the eigenvalue problem. Once the concentrated mass is off the center, Eqs. (10) and (11) can no longer be valid. Even when the concentrated mass at the center, the above formulation by Ilic et al. [9] cannot be applied to find the system eigenfrequency of even modes. Because the even modes of a clamped-clamped beam are antisymmetric, the symmetry conditions of Eqs. (10) and (11) are also invalid. The characteristic equation of Eq. (12) can only be used to find the eigenfrequencies of the odd (symmetric) modes when the concentrated is located at the center. The above approach of dividing the domain into $r+s+1$ subdomains is the generalized one, which is capable of computing all the eigenfrequencies with different concentrated mass/spring locations.

Here it is also noteworthy to discuss and compare the "exact approach" presented by $\mathrm{Li}$ and his colleagues [34,35] with the above analytical method. Although Li et al. [34] computed the eigenfrequencies of the rods longitudinal vibrations coupled by concentrated translational springs and $\mathrm{Li}[35]$ is on the eigenfrequencies of rectangular plate with line-concentrated mass and elastic support, Li's approach can be applied to the above eigenfrequency problem of the transverse vibration of beam carrying concentrated mass/spring. Li's approach $[34,35]$ can be summarized as follows: Instead of writing the general solution forms such as Eq. (5), Li used the matching conditions similar to Eqs. (6) and (7) to construct the delicate solution forms before and after the concentrated masses/springs locations. Li's approach in essence is the same one as the above analytical method. However, with Li's method of constructing the solution forms, the number of the unknown constants in Eq. (5) will be significantly reduced. Li's formulation of the eigenvalue problem is thus expected to have much less computation and the trade-off is the complex derivation of solution forms.

2.2 Galerkin Method. Unlike the analytical method, Galerkin method uses the Dirac delta function to directly incorporate the effects of concentrated mass and spring into the governing equation. For the sake of brevity, the governing equation is given as follows, which is similar to those in Refs. [27,28]:

$$
m \frac{\partial^{2} w}{\partial t^{2}}+\sum_{e=1}^{r} M_{e} \delta\left(x-u_{e}\right) \frac{\partial^{2} w}{\partial t^{2}}+E I \frac{\partial^{4} w}{\partial x^{4}}+\sum_{e=1}^{s} K_{e} \delta\left(x-v_{e}\right) w=0
$$

Again, $M_{e}$ and $u_{e}(e=1-r)$ are the concentrated mass and its coordinate, respectively. $K_{e}$ and $v_{e}(e=1-s)$ are the concentrated spring stiffness and its coordinate, respectively. $\delta$ here is the Dirac 
delta function. With the same nondimensionalization scheme of Eq. (2), Eq. (13) is now nondimensionalized as follows:

$$
\left[1+\sum_{e=1}^{r} \alpha_{e} \delta\left(\xi-\mu_{e}\right)\right] \frac{\partial^{2} W}{\partial \tau^{2}}+\frac{\partial^{4} W}{\partial \xi^{4}}+\sum_{e=1}^{s} \beta_{e} \delta\left(\xi-\nu_{e}\right) W=0
$$

$W(\xi, \tau)$ is assumed to have the following form:

$$
W(\xi, \tau)=\sum_{j=1}^{N} a_{j} \phi_{j}(\xi) e^{i \omega \tau}
$$

$N$ is the mode number and $a_{j}$ is the unknown constant (modal amplitude). $\phi_{j}$ is the $j$ th mode shape of a uniform beam given as follows [36]:

$$
\phi_{j}(\xi)=A_{j} \sin \left(\kappa_{j} \xi\right)+B_{j} \cos \left(\kappa_{j} \xi\right)+C_{j} \sinh \left(\kappa_{j} \xi\right)+D_{j} \cosh \left(\kappa_{j} \xi\right)
$$

$\kappa_{j}, A_{j}, B_{j}, C_{j}$, and $D_{j}$ are the constants given by Chang and Craig [36] for the $j$ th mode shape of a uniform beam and they vary with different boundary conditions. $\kappa_{j}^{2}$ is the dimensionless $j$ th eigenfrequency of a uniform beam. $\phi_{j}$ is orthogonal [21]. Because some of mode shapes given by Chang and Craig are not normal, $A_{j}, B_{j}, C_{j}$, and $D_{j}$ are divided by $\sqrt{\int_{0}^{1} \phi_{j}^{2}(\xi) d \xi}$ to be normalized for later computation and statement convenience. Therefore, $\phi_{j}$ has the following orthonormal property:

$$
\int_{0}^{1} \phi_{i}(\xi) \phi_{j}(\xi) d \xi=\delta_{i j}
$$

$\delta_{i j}$ here is the Kronecker delta function. Substitute Eq. (15) into Eq. (14), times $\phi_{i}(\xi)(i=1-N)$, and integrate, the following equation is obtained:

$$
\begin{aligned}
& -\omega^{2} \int_{0}^{1} \phi_{i}(\xi)\left[1+\sum_{e=1}^{r} \alpha_{e} \delta\left(\xi-\mu_{e}\right)\right] \sum_{j=1}^{N} a_{j} \phi_{j}(\xi) d \xi \\
& +\int_{0}^{1} \phi_{i}(\xi)\left[\kappa_{j}^{4}+\sum_{e=1}^{s} \beta_{e} \delta\left(\xi-\nu_{e}\right)\right] \sum_{j=1}^{N} a_{j} \phi_{j}(\xi) d \xi=0
\end{aligned}
$$

With the use of the integration property of the Dirac delta function [21,27,28,32], Eq. (18) can also be written in the following form:

$$
G A=0
$$

$A^{T}=\left(a_{1}, a_{2}, \ldots, a_{N}\right), G$ is an $N \times N$ matrix and each of its elements is defined as follows:

$$
G_{i j}=\kappa_{j}^{4} \delta_{i j}+\sum_{e=1}^{s} \beta_{e} \phi_{i}\left(\nu_{e}\right) \phi_{j}\left(\nu_{e}\right)-\omega^{2}\left[\delta_{i j}+\sum_{e=1}^{r} \alpha_{e} \phi_{i}\left(\mu_{e}\right) \phi_{j}\left(\mu_{e}\right)\right]
$$

To have nontrivial solution of $A$, the determinant of $G$, $\operatorname{det}(G)$ must be set zero, which results in the characteristic equation of $\omega$. So the eigenfrequency computation of the Galerkin method depends on the mode number $N$. Because the concentrated mass/ spring can severely distort the mode shapes of system, large number of modes may be required to approximate such distortion. If the concentrated mass/spring is small and the system $j$ th mode shape is not severely distorted, which means one $j$ th mode of a uniform beam can have a good approximation on the $j$ th mode shape of the system with concentrated mass/spring. The following close form approximation of the $j$ th eigenfrequency is derived from Eq. (20) by setting diagonal term $G_{j j}=0$ :

$$
\omega_{j}=\sqrt{\frac{\kappa_{j}^{4}+\sum_{e=1}^{s} \beta_{e} \phi_{j}^{2}\left(\nu_{e}\right)}{1+\sum_{e=1}^{r} \alpha_{e} \phi_{j}^{2}\left(\mu_{e}\right)}}
$$

When $\beta_{e}=\alpha_{e}=0$, Eq. (21) results in $\omega_{j}=\kappa_{j}^{2}$, which recovers the $j$ th eigenfrequency of a uniform beam. When $\beta_{e}=0$ and $\alpha_{e} \neq 0$, it is the case of no spring and Eq. (21) shares the same square root form of the eigenfrequency approximation expression presented in Ref. [9].

2.3 Finite Mode Transform Method. For the FMTM, the governing equation is still Eq. (14). $W$ is now assumed to have the following form:

$$
W(\xi, \tau)=\psi(\xi) e^{i \omega \tau}
$$

Compared with Eq. (15), it is not hard to find out that

$$
\psi(\xi)=\sum_{j=1}^{N} a_{j} \phi_{j}(\xi)
$$

Similarly, substitute Eq. (22) into Eq. (14), times $\phi_{i}$, and integrate, the following equation is obtained:

$$
\begin{gathered}
\int_{0}^{1} \phi_{i}(\xi)\left\{\frac{\partial^{4} \psi(\xi)}{\partial \xi^{4}}-\omega^{2} \psi(\xi)+\left[\sum_{e=1}^{s} \beta_{e} \delta\left(\xi-\nu_{e}\right)-\omega^{2} \sum_{e=1}^{r} \alpha_{e} \delta(\xi\right.\right. \\
\left.\left.\left.-\mu_{e}\right)\right] \psi(\xi)\right\} d \xi=0
\end{gathered}
$$

substitute Eq. (23) into the first two terms of Eq. (24) and $\psi(\xi)$ in the last two terms associated with the Dirac delta function keep unsubstituted. By using the orthonormality of mode shape $\phi$ and the integration property of Dirac delta function again, $a_{j}$ is found as follows:

$$
a_{j}=\frac{\omega^{2} \sum_{e=1}^{r} \alpha_{e} \phi_{j}\left(\mu_{e}\right) \psi\left(\mu_{e}\right)-\sum_{e=1}^{s} \beta_{e} \phi_{j}\left(\nu_{e}\right) \psi\left(\nu_{e}\right)}{\kappa_{j}^{4}-\omega^{2}}
$$

Now substitute $a_{j}$ of Eq. (25) into Eq. (23), $\psi$ is rewritten as follows:

$$
\psi(\xi)=\sum_{e=1}^{r} H_{e}(\omega, \xi) \psi\left(\mu_{e}\right)-\sum_{e=1}^{s} I_{e}(\omega, \xi) \psi\left(\nu_{e}\right)
$$

$H_{e}(\omega, \xi)$ and $I_{e}(\omega, \xi)$ are defined as follows:

$$
H_{e}(\omega, \xi)=\frac{\omega^{2} \sum_{j=1}^{N} \alpha_{e} \phi_{j}\left(\mu_{e}\right) \phi_{j}(\xi)}{\kappa_{j}^{4}-\omega^{2}}, \quad I_{e}(\omega, \xi)=\frac{\sum_{j=1}^{N} \beta_{e} \phi_{j}\left(\nu_{e}\right) \phi_{j}(\xi)}{\kappa_{j}^{4}-\omega^{2}}
$$

Because Eq. (26) is valid for any $\xi$, let $\xi=\mu_{e}(e=1$ to $r)$ and $\xi$ $=\nu_{e}(e=1$ to $s)$, the following set of $r+s$ equations is obtained: 


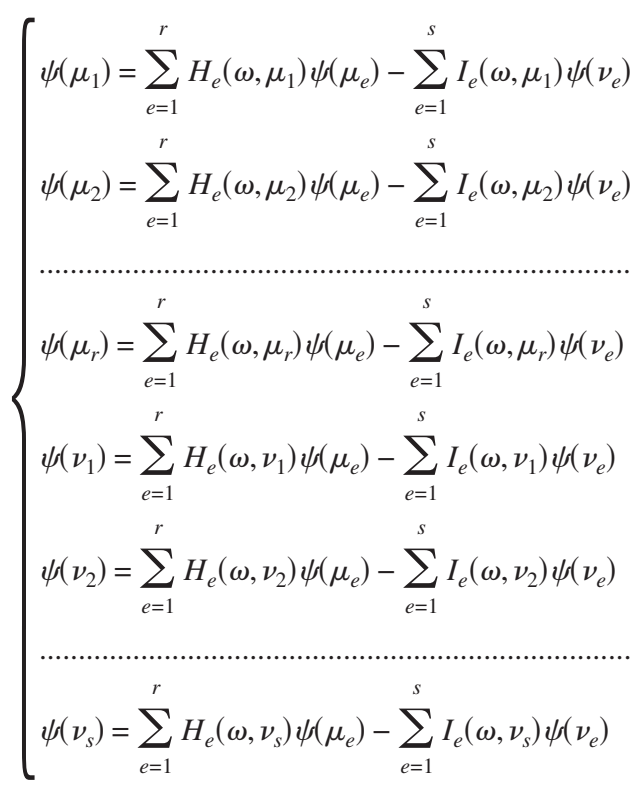

Equation (28) can also be rewritten as the following form by simple manipulation:

$$
C V=0
$$

$V$ is an $r+s$ vector and $V^{T}=\left[\psi\left(\mu_{1}\right), \psi\left(\mu_{2}\right), \ldots, \psi\left(\mu_{r}\right), \psi\left(\nu_{1}\right), \psi\left(\nu_{2}\right), \ldots, \psi\left(\nu_{s}\right)\right] . C$ is an $(r+s) \times(r+s)$ matrix and $C=C(\omega)$. $C(\omega)$ is defined as follows:

$$
C(\omega)=\left(\begin{array}{ccccccc}
H_{1}\left(\omega, \mu_{1}\right)-1 & H_{2}\left(\omega, \mu_{1}\right) & \ldots & H_{r}\left(\omega, \mu_{1}\right) & -I_{1}\left(\omega, \mu_{1}\right) & \ldots & -I_{s}\left(\omega, \mu_{1}\right) \\
H_{1}\left(\omega, \mu_{2}\right) & H_{2}\left(\omega, \mu_{2}\right)-1 & \ldots & H_{r}\left(\omega, \mu_{2}\right) & -I_{1}\left(\omega, \mu_{2}\right) & \ldots & -I_{s}\left(\omega, \mu_{2}\right) \\
\ldots & \ldots & \ldots & \ldots & \ldots & \ldots & \ldots \\
H_{1}\left(\omega, \mu_{r}\right) & H_{2}\left(\omega, \mu_{r}\right) & \ldots & H_{r}\left(\omega, \mu_{r}\right)-1 & -I_{1}\left(\omega, \mu_{r}\right) & \ldots & -I_{s}\left(\omega, \mu_{r}\right) \\
H_{1}\left(\omega, \nu_{1}\right) & H_{2}\left(\omega, \nu_{1}\right) & \ldots & H_{r}\left(\omega, \nu_{1}\right) & -I_{1}\left(\omega, \nu_{1}\right)-1 & \ldots & -I_{s}\left(\omega, \nu_{1}\right) \\
\ldots & \ldots & \ldots & \ldots & \ldots & \ldots & \ldots \\
H_{1}\left(\omega, \nu_{s}\right) & H_{2}\left(\omega, \nu_{s}\right) & \ldots & H_{r}\left(\omega, \nu_{s}\right) & -I_{1}\left(\omega, \nu_{s}\right) & \ldots & -I_{s}\left(\omega, \nu_{s}\right)-1
\end{array}\right)
$$

As far as not all of $\mu_{e}(e=1$ to $r)$ and $\nu_{e}(e=1$ to $s)$ are on the beam nodes, which means that $V$ has a nontrivial solution, the determinant of $C$ must be zero. Therefore, $\operatorname{det}(C)=0$ is the characteristic equation to determine the eigenfrequency $\omega$ of the beam with concentrated masses and springs. $\operatorname{det}(C)=0$ is a polynomial equation of $\omega$. Clearly, here the eigenfrequency computation by FMTM formulation is an eigenvalue problem of an $(r+s) \times(r$ $+s)$ matrix in comparison with the eigenvalue problem of a $4(r$ $+s+1) \times 4(r+s+1)$ matrix by the analytical method formulation.

2.4 Computation Example and Discussion. In order to compare our results with Low's [22], the computation example of a beam with only one concentrated mass is presented. Instead of $\omega_{j}$ (the $j$ th eigenfrequency of the system with the concentrated mass), $\Lambda_{j}=\sqrt{\omega_{j}}$ is used in the following two tables. In this computation example, the location of the concentrated mass is fixed at $\mu_{1}$ $=0.1$ and the beam is clamped at the both ends. In the following two tables, the $\Lambda_{1}$ values of analytical method are all taken from Low's paper [22]. For both the Galerkin method and FMTM, Newton-Rhapson method [37] is used to solve the characteristic equations. Because the $\Lambda_{j}$ values obtained by the analytical method have been shown accurate as compared with the experimental data [22], $\Lambda_{1}^{\mathrm{FMTM}}-\Lambda_{1}^{\text {analytical }} / \Lambda_{1}^{\text {analytical }}$ is introduced to indicate the $\Lambda_{1}$ error computed by FMTM. Figure 2 shows the error convergence of $\alpha_{1}=20$ and 100 as the function of mode number

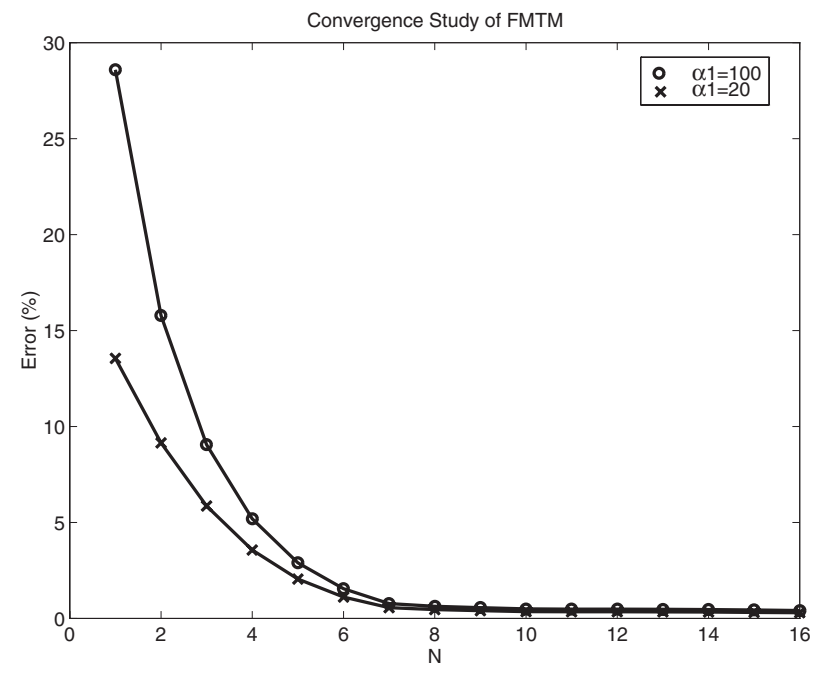

Fig. 2 The convergence study on FMTM computation on the fundamental frequency of the beam with a concentrated mass as the mode number increases. Two cases of $\alpha_{1}=20$ and $\alpha_{1}$ $=100$ are presented and the concentrated mass is located at $\mu_{1}=0.1$. 
Table 1 Comparison of $\Lambda_{1}$ computed by the analytical method and FMTM

\begin{tabular}{|c|c|c|c|c|c|c|c|c|c|c|}
\hline Methods / $\alpha_{1}$ & 0.5 & 1.0 & 1.5 & 2.0 & 5.0 & 10.0 & 20.0 & 50.0 & 80.0 & 100.0 \\
\hline Analytical (Ref. [22]) & 4.708 & 4.685 & 4.66 & 4.634 & 4.451 & 4.123 & 3.64 & 2.969 & 2.654 & 2.515 \\
\hline FMTM & 4.708 & 4.685 & 4.66 & 4.634 & 4.454 & 4.132 & 3.653 & 2.982 & 2.667 & 2.527 \\
\hline
\end{tabular}

$(N)$. The errors of $\alpha_{1}=100$ are always larger than those of $\alpha_{1}$ $=20$ and they both rapidly reduce with the increase of the mode number. When $N \geq 10$, both errors are less than $0.5 \%$.

In Table 1 , ten modes $(N=10)$ are used in FMTM. $\Lambda_{1}$ s obtained by the analytical method and FMTM agree well with each other. Unfortunately, FMTM presented here does not yield accurate results for $\alpha_{1}<0.5$. The reason that FMTM has the numerical difficulty of finding accurate solutions for small $\alpha_{1}$ is given as follows. For the beam with a single concentrated mass $\alpha_{1}$ at $\mu_{1}$, the following characteristic equation can be derived from either Eq. (28) or Eq. (30):

$$
\sum_{j=1}^{N} \frac{\alpha_{1} \phi_{j}^{2}\left(\mu_{1}\right) \omega^{2}}{\kappa_{j}^{4}-\omega^{2}}=1
$$

When the concentrated mass is small $\left(\alpha_{1} \approx 0\right)$ or its location close to a node or a clamped/hinged end $\left(\phi_{j}\left(\mu_{1}\right) \approx 0\right), \omega$ is expected to be $\omega \approx \kappa_{j}^{2}\left(\kappa_{j}^{2}\right.$ is the $j$ th eigenfrequency of a uniform beam [36]); therefore, the leftside of Eq. (31) is a 0/0 type of limit, which approaches 1. However, numerically it is difficult for FMTM to find $\omega$ solution around $\kappa_{j}^{2}$ accurately under this $0 / 0$ scenario though the algorithm used here has a mechanism of precision control [37]. When the concentrated mass is large or its location is away from node or clamped/hinged end, which causes the system eigenfrequencies to shift significantly from those of a uniform beam, FMTM is fairly accurate, as demonstrated in Table 1 for large $\alpha_{1}$ cases.

Table 2 shows the comparison of $\Lambda_{1}$ s obtained by the analytical method and Galerkin method with different mode numbers. When the concentrated mass is small (or close to the nodes), compared with FMTM, the Galerkin method has no problem of computing the eigenfrequency and show good accuracy. However, when the concentrated mass is large, the Galerkin method shows significant error compared with the values obtained by the analytical method. For the Galerkin method of $N=1$, the error becomes larger with the increase of $\alpha_{1}$. The error starts at $\alpha_{1}=1$ and becomes very significant when $\alpha_{1}=20$. Physically, the Galerkin method of $N$ $=1$ is to use the first mode shape of a uniform beam to approximate the deflection curve of the beam with concentrated mass. Like the Rayleigh method, the accuracy of the Galerkin method also depends on the how close the approximation curve is. With the increase of concentrated mass, the approximation by the first mode shape of a uniform beam will deviate more and more from the beam real defection curve of the beam, as demonstrated in Fig. 3 . Figure 3 plots the first and second mode shapes of $\alpha_{1}=0$ (uniform beam), $\alpha_{1}=1$ and 20. The single concentrated mass is also fixed at $\xi=\mu_{1}=0.1$. The first mode shape of $\alpha_{1}=1$ is not significantly different from that of $\alpha_{1}=0$ and this explains why the $\Lambda_{1}$ error of $\alpha_{1}=1$ in Table 2 is relatively small. But the second mode shape difference between $\alpha_{1}=1$ and $\alpha_{1}=0$ is very significant. This fact has the following implication: When the concentrated mass or spring is large, Eq. (21) of the Galerkin method using one mode approximation cannot be accurate because of the severe distortion of the system mode shapes. The first mode shape of $\alpha_{1}=20$ is so severely distorted that it looks more like the second mode shape of a uniform beam. This explains why the Galerkin method of $N=1$ cannot accurately compute $\Lambda_{1}$ at $\alpha_{1}=20$. When $N=2$, the first and second modes of a uniform beam are used to approximate the real deflection of the $\alpha_{1}=20$ case, and much accurate result is obtained, as shown in Table 2. However, it is also noticed that in Table 2, increasing mode number $N$ does not necessarily improve the accuracy, especially for large $\alpha_{1}$ cases. The similar scenario is also observed by Senba and Furuya [38] that increasing the number of mode shape may lead to even larger error and their explanation is the same as the above one: difference between the real deflection and the approximation function of mode shape. In the computation sense, the reason may lie in the matrix element expression of Galerkin method in Eq. (20). For the single concentrated mass case, Eq. (20) is written as $G_{i j}=\kappa_{j}^{4} \delta_{i j}-\omega^{2}\left[\delta_{i j}\right.$ $\left.+\alpha_{1} \phi_{i}\left(\mu_{1}\right) \phi_{j}\left(\mu_{1}\right)\right]$. From this expression, it is noticed that when $\alpha_{1}$ is small, the diagonal terms of the matrix is large/dominant compared with the off-diagonal terms and Galerkin method shows good accuracy. With the increase of $\alpha_{1}$, the magnitude of the off-diagonal terms $G_{i j}=-\omega^{2} \alpha_{1} \phi_{i}\left(\mu_{1}\right) \phi_{j}\left(\mu_{1}\right)(i \neq j)$ increases and the diagonal terms $G_{i i}=\kappa_{i}^{4}-\omega^{2}\left[1+\alpha_{1} \phi_{i}^{2}\left(\mu_{1}\right)\right]$ decreases, which may result in the ill-conditioning of the matrix and lead to the larger computation error. The Galerkin method uses the mode shapes defined by Eq. (16), which is the eigenfunctions of a uniform beam, to formulate the eigenvalue problem. It is worth pointing that sometimes the eigenfrquency computation formulated by eigenfunctions may have "an intolerably slow convergence rate" [39].

Because the resonator mass sensor excited with higher resonant frequency shows much better sensitivity of detecting tiny mass change $[11,12]$, it is necessary to emphasize that though only the fundamental eigenfrequency is listed and compared in Tables 1 and 2 , the three methods above are all capable of computing the eigenfrequencies of higher modes. In the resonator mass sensor application, the ambient environment has significant influence on the system parameters. For example, the system mass and damping may change with pressure; the material Young's modulus may change with the temperature [40]. How to specify the mass and Young's modulus change is given by Sandberg et al. [40]. Here let us have a brief discussion on the damping issue. Damping (or, say, the quality factor which is inversely proportional to damping) has dramatic impact on the sensitivity of the resonator mass sensor $[11,12]$. It is noticed that Eq. (1) has no damping term. The reason to omit the damping term in this paper is purely in order to have an apple-to-apple comparison with those formulations and results obtained in Refs. [9,22], which do not include the damping term.

Table 2 Comparison of $\Lambda_{1}$ computed by analytical method and Galerkin method with different modes

\begin{tabular}{|c|c|c|c|c|c|c|c|c|c|c|c|}
\hline Methods $/ \alpha_{1}$ & 0.01 & 0.05 & 0.1 & 0.2 & 0.3 & 0.4 & 1.0 & 1.5 & 2.0 & 10.0 & 20.0 \\
\hline Analytical (Ref. [22]) & 4.73 & 4.728 & 4.726 & 4.721 & 4.717 & 4.713 & 4.685 & 4.66 & 4.634 & 4.123 & 3.64 \\
\hline Galerkin $(N=1)$ & 4.73 & 4.728 & 4.726 & 4.721 & 4.717 & 4.713 & 4.689 & 4.667 & 4.649 & 4.382 & 4.133 \\
\hline Galerkin $(N=2)$ & 4.73 & 4.728 & 4.726 & 4.721 & 4.717 & 4.713 & 4.684 & 4.659 & 4.632 & 4.149 & 3.707 \\
\hline Galerkin $(N=3)$ & 4.73 & 4.728 & 4.726 & 4.721 & 4.717 & 4.71 & 4.668 & 4.622 & 4.568 & 3.693 & 3.176 \\
\hline Galerkin $(N=4)$ & 4.73 & 4.728 & 4.725 & 4.72 & 4.712 & 4.703 & 4.622 & 4.52 & 4.402 & 3.261 & 2.77 \\
\hline Galerkin $(N=5)$ & 4.73 & 4.728 & 4.724 & 4.716 & 4.704 & 4.689 & 4.526 & 4.337 & 4.153 & 2.936 & 2.482 \\
\hline
\end{tabular}



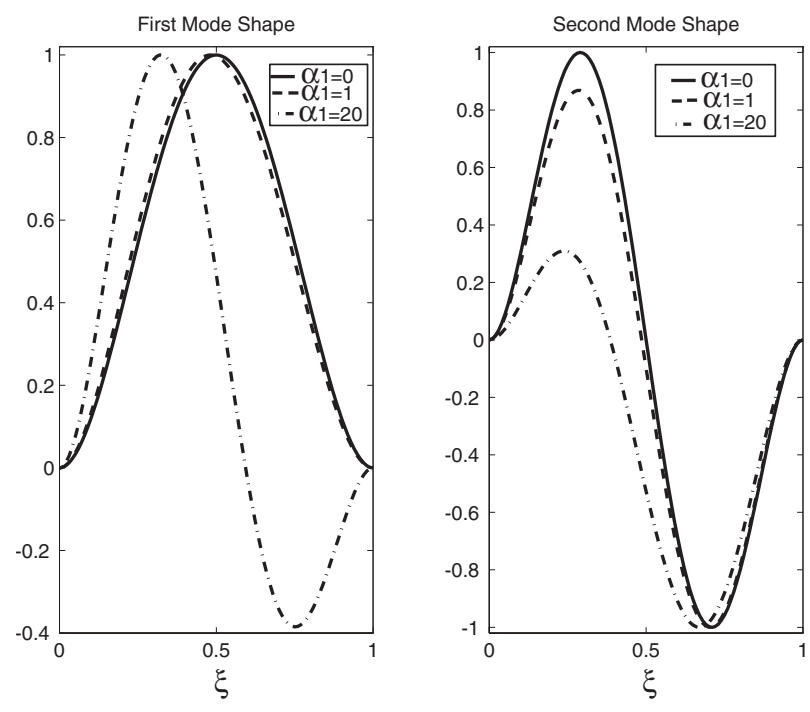

Fig. 3 The first and second mode shapes of the beam with a concentrated mass when $\alpha_{1}$ is taken as 0,1 , and 20, respectively. The concentrated mass is also located at $\mu_{1}=0.1$.

Although the governing equation of Jin et al. [12] has the damping term, their Eq. (4) of the eigenfrequency expression actually has no damping influence. For the analytical method presented in this paper, it will be of great difficulty to incorporate the damping effect if not impossible. For the Galerkin method, with the introduction of the system damping (or more complex gyroscopic term), there will be some change of the eigenfrequency computation formulation and the detailed formulation can be found in Refs. $[27,41]$. For FMTM, it is very easy to incorporate the damping effect. The following outlines the procedures.

With the introduction of damping, Eq. (14) now becomes as the following:

$$
\left[1+\sum_{e=1}^{r} \alpha_{e} \delta\left(\xi-\mu_{e}\right)\right] \frac{\partial^{2} W}{\partial \tau^{2}}+c \frac{\partial W}{\partial \tau}+\frac{\partial^{4} W}{\partial \xi^{4}}+\sum_{e=1}^{s} \beta_{e} \delta\left(\xi-\nu_{e}\right) W=0
$$

Here $c \partial W / \partial \tau$ is the damping term and $c$ is a dimensionless damping coefficient. Now the Eq. (24) also changes as the following

$$
\begin{gathered}
\int_{0}^{1} \phi_{i}(\xi)\left\{\frac{\partial^{4} \psi}{\partial \xi^{4}}+i c \omega \psi-\omega^{2} \psi+\left[\sum_{e=1}^{s} \beta_{e} \delta\left(\xi-\nu_{e}\right)-\omega^{2} \sum_{e=1}^{r} \alpha_{e} \delta(\xi\right.\right. \\
\left.\left.\left.-\mu_{e}\right)\right] \psi(\xi)\right\} d \xi=0
\end{gathered}
$$

Therefore, the previous $a_{j}$ given by Eq. (25) is now changed as follows:

$$
a_{j}=\frac{\omega^{2} \sum_{e=1}^{r} \alpha_{e} \varphi_{j}\left(\mu_{e}\right) \psi\left(\mu_{e}\right)-\sum_{e=1}^{s} \beta_{e} \varphi_{j}\left(\nu_{e}\right) \psi\left(\nu_{e}\right)}{\kappa_{j}^{4}+i c \omega-\omega^{2}}
$$

Then follow all the steps from Eq. (26) to Eq. (30) and new eigenfrequencies containing damping effect can be obtained.

\section{FSTM on the Plate With Four Edges Simply Sup- ported Carrying Arbitrary Number of Concentrated Mass and Spring}

3.1 Problem Formulation. For the sake of brevity, the governing equation for a rectangular plate carrying arbitrarily distributed concentrated masses and springs is directly given as follows:

$$
\begin{gathered}
D \nabla^{4} w+\left[M+\sum_{i=1}^{r} M_{i} \delta\left(x-u_{i}\right) \delta\left(y-v_{i}\right)\right] w_{t t} \\
+\sum_{i=1}^{s} K_{i} \delta\left(x-a_{i}\right) \delta\left(y-b_{i}\right) w=0
\end{gathered}
$$

$w$ is the plate transverse displacement and $w_{t t}=\partial^{2} W / \partial t^{2} . \delta$ is still the Dirac delta function. $\nabla^{4}$ is the operator defined as $\nabla^{4}$ $=\left(\partial^{4} / \partial x^{4}\right)+2\left(\partial^{4} / \partial x^{2} \partial y^{2}\right)+\left(\partial^{4} / \partial y^{4}\right) . M$ and $D$ are the mass per unit area of the plate and the plate flexural rigidity, respectively. $D=E h^{3} / 12\left(1-\nu^{2}\right)$ ( $h$ is the plate thickness. $E$ and $\nu$ are the plate Young's modulus and Poisson's ratio, respectively.) There are $r$ concentrated masses; $M_{i}$ (i=1 to $r$ ) is the concentrated mass; $u_{i}$ and $v_{i}$ are the corresponding coordinates. There are $s$ concentrated translational springs; $K_{i}(i=1$ to $s)$ is the stiffness of concentrated spring; $a_{i}$ and $b_{i}$ are the corresponding coordinates. The plate is isotropic and homogeneous. Equation (35) does not include the concentrated mass effect of moment of inertia [32], either.

Similarly, the plate transverse displacement $w$ is assumed to have the following solution form:

$$
w=\psi(x, y) e^{i \omega t}
$$

Substitute Eq. (36) into Eq. (35), the following equation is obtained:

$$
\begin{aligned}
\nabla^{4} \psi- & \frac{\omega^{2}}{D}\left[M+\sum_{i=1}^{r} M_{i} \delta\left(x-u_{i}\right) \delta\left(y-v_{i}\right)\right] \psi+\frac{1}{D} \sum_{i=1}^{s} K_{i} \delta\left(x-a_{i}\right) \delta(y \\
\left.-b_{i}\right) \psi & =0
\end{aligned}
$$

$\psi(x, y)$ is the spatial part solution of the plate carrying concentrated masses and springs. For the plate with four edges simply supported, $\psi(x, y)$ can be approximated by the following series [29]:

$$
\psi(x, y)=\frac{4}{a b} \sum_{m=1}^{\infty} \sum_{n=1}^{\infty} \Omega_{m n} \sin \left(\frac{m \pi}{a} x\right) \sin \left(\frac{n \pi}{b} y\right)
$$

Equation (38) is a double Fourier expansion of $\psi(x, y)$ and $\Omega_{m n}$ is thus defined as

$$
\Omega_{m n}=\int_{0}^{a} \int_{0}^{b} \psi(x, y) \sin \left(\frac{m \pi}{a} x\right) \sin \left(\frac{n \pi}{b} y\right) d x d y
$$

$a$ and $b$ are the plate length and width, respectively. $\sin ((m \pi / a) x) \sin ((n \pi / b) y)$ is the $m-n$th mode shape of a uniform plate with four edges simply supported.

Times equation (37) with $\sin ((m \pi / a) x) \sin ((n \pi / b) y)$ and have a double integral operation, the following equation is derived

$$
\begin{aligned}
\int_{0}^{a} \int_{0}^{b}\left\{\nabla^{4} \psi-\frac{\omega^{2}}{D}\left[M+\sum_{i=1}^{r} M_{i} \delta\left(x-u_{i}\right) \delta\left(y-v_{i}\right)\right] \psi\right. \\
\left.+\frac{1}{D} \sum_{i=1}^{s} K_{i} \delta\left(x-a_{i}\right) \delta\left(y-b_{i}\right) \psi\right\} \sin \left(\frac{m \pi}{a} x\right) \sin \left(\frac{n \pi}{b} y\right) d x d y=0
\end{aligned}
$$

Similarly, substitute the $\psi(x, y)$ expression of Eq. (38) into Eq. (40) except the terms related to the concentrated masses and springs. Again, by using integration by parts, the boundary conditions, integration property of the Dirac delta function, and orthogonality of sine functions, the following equation is derived from Eq. (40): 


$$
\pi^{4}\left(\frac{m^{2}}{a^{2}}+\frac{n^{2}}{b^{2}}\right)^{2} \Omega_{m n}-\frac{M \omega^{2}}{D} \Omega_{m n}-\frac{\omega^{2}}{D} \sum_{i=1}^{r} M_{i} \psi\left(u_{i}, v_{i}\right) \sin \left(\frac{m \pi}{a} u_{i}\right) \sin \left(\frac{n \pi}{b} v_{i}\right)+\frac{1}{D} \sum_{i=1}^{s} K_{i} \psi\left(a_{i}, b_{i}\right) \sin \left(\frac{m \pi}{a} a_{i}\right) \sin \left(\frac{n \pi}{b} b_{i}\right)=0
$$

From Eq. (41), $\Omega_{m n}$ is solved as

$$
\Omega_{m n}=\frac{\frac{\omega^{2}}{D} \sum_{i=1}^{r} M_{i} \psi\left(u_{i}, v_{i}\right) \sin \left(\frac{m \pi}{a} u_{i}\right) \sin \left(\frac{n \pi}{b} v_{i}\right)-\frac{1}{D} \sum_{i=1}^{s} K_{i} \psi\left(a_{i}, b_{i}\right) \sin \left(\frac{m \pi}{a} a_{i}\right) \sin \left(\frac{n \pi}{b} b_{i}\right)}{\pi^{4}\left(\frac{m^{2}}{a^{2}}+\frac{n^{2}}{b^{2}}\right)^{2}-\frac{M \omega^{2}}{D}}
$$

Substitute Eq. (42) into Eq. (38), $\psi(x, y)$ is now rewritten as follows:

$$
\psi(x, y)=\sum_{i=1}^{r} A_{i}(\omega, x, y) \psi\left(u_{i}, v_{i}\right)-\sum_{i=1}^{s} B_{i}(\omega, x, y) \psi\left(a_{i}, b_{i}\right)
$$

$A_{i}$ and $B_{i}$ are defined as follows:

$$
\begin{aligned}
& A_{i}(\omega, x, y) \\
& =\frac{4 \omega^{2} M_{i}}{a b D} \sum_{m=1}^{\infty} \sum_{n=1}^{\infty} \frac{\sin \left(\frac{m \pi}{a} u_{i}\right) \sin \left(\frac{n \pi}{b} v_{i}\right) \sin \left(\frac{m \pi}{a} x\right) \sin \left(\frac{n \pi}{b} x\right)}{\pi^{4}\left(\frac{m^{2}}{a^{2}}+\frac{n^{2}}{b^{2}}\right)^{2}-\frac{M \omega^{2}}{D}} \\
& B_{i}(\omega, x, y) \\
& =\frac{4 K_{i}}{a b D} \sum_{m=1}^{\infty} \sum_{n=1}^{\infty} \frac{\sin \left(\frac{m \pi}{a} a_{i}\right) \sin \left(\frac{n \pi}{b} b_{i}\right) \sin \left(\frac{m \pi}{a} x\right) \sin \left(\frac{n \pi}{b} x\right)}{\pi^{4}\left(\frac{m^{2}}{a^{2}}+\frac{n^{2}}{b^{2}}\right)^{2}-\frac{M \omega^{2}}{D}}
\end{aligned}
$$

Equation (43) is valid for any $x, y$, so substitute $u_{i}, v_{i}(i=1, r)$ and $a_{i}, b_{i}(i=1, s)$ into Eq. (43). Therefore, the following set of $r+s$ equations are obtained.

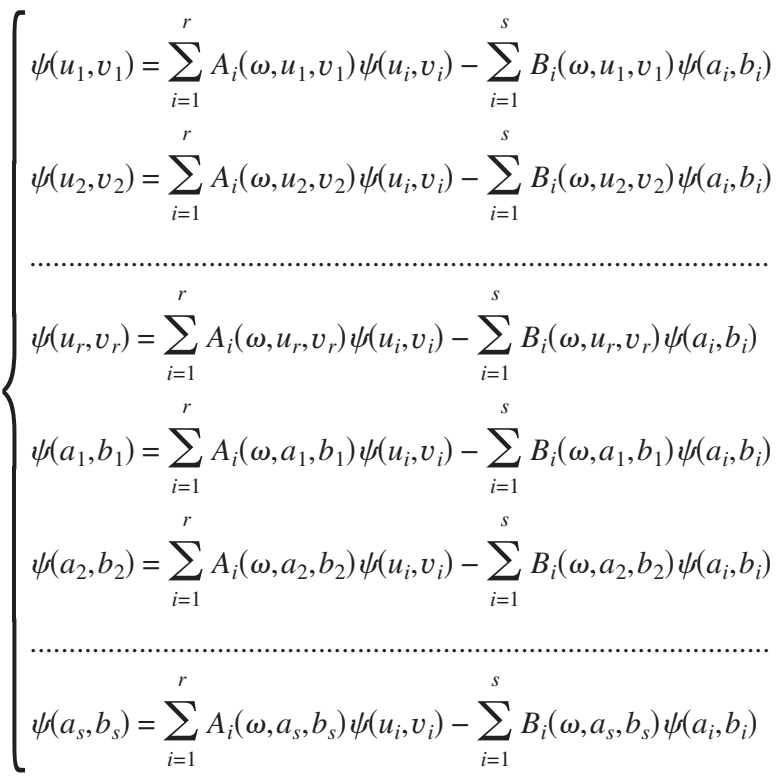

Equation (45) can also be rewritten as the following form by simple manipulation:

$$
C V=0
$$

$C$ is a $(r+s) \times(r+s)$ matrix and $C=C(\omega) . C(\omega)$ is defined as follows:

$$
C(\omega)=\left(\begin{array}{ccccccc}
A_{1}\left(\omega, u_{1}, v_{1}\right)-1 & A_{2}\left(\omega, u_{1}, v_{1}\right) & \ldots & A_{r}\left(\omega, u_{1}, v_{1}\right) & -B_{1}\left(\omega, u_{1}, v_{1}\right) & \ldots & -B_{s}\left(\omega, u_{1}, v_{1}\right) \\
A_{1}\left(\omega, u_{2}, v_{2}\right) & A_{2}\left(\omega, u_{2}, v_{2}\right)-1 & \ldots & A_{r}\left(\omega, u_{2}, v_{2}\right) & -B_{1}\left(\omega, u_{2}, v_{2}\right) & \ldots & -B_{s}\left(\omega, u_{2}, v_{2}\right) \\
\ldots & \ldots & \ldots & \ldots & \ldots & \ldots & \ldots \\
A_{1}\left(\omega, u_{r}, v_{r}\right) & A_{2}\left(\omega, u_{r}, v_{r}\right) & \ldots & A_{r}\left(\omega, u_{r}, v_{r}\right)-1 & -B_{1}\left(\omega, u_{r}, v_{r}\right) & \ldots & -B_{s}\left(\omega, u_{r}, v_{r}\right) \\
A_{1}\left(\omega, a_{1}, b_{1}\right) & A_{2}\left(\omega, a_{1}, b_{1}\right) & \ldots & A_{r}\left(\omega, a_{1}, b_{1}\right) & -B_{1}\left(\omega, a_{1}, b_{1}\right)-1 & \ldots & -B_{s}\left(\omega, a_{1}, b_{1}\right) \\
\ldots & \ldots & \ldots & \ldots & \ldots & \ldots & \ldots \\
A_{1}\left(\omega, a_{s}, b_{s}\right) & A_{2}\left(\omega, a_{s}, b_{s}\right) & \ldots & A_{r}\left(\omega, a_{s}, b_{s}\right) & -B_{1}\left(\omega, a_{s}, b_{s}\right) & \ldots & -B_{s}\left(\omega, a_{s}, b_{s}\right)-1
\end{array}\right)
$$

$V$ is a $r+s$ vector and $V^{T}=\left[\psi\left(u_{1}, v_{1}\right), \psi\left(u_{2}, v_{2}\right), \ldots, \psi\left(u_{r}, v_{r}\right)\right.$, $\left.\psi\left(a_{1}, b_{1}\right), \psi\left(a_{2}, b_{2}\right), \ldots, \psi\left(a_{s}, b_{s}\right)\right]$. Again, as far as not all of $\left(u_{i}, v_{i}\right)$ and $\left(a_{i}, b_{i}\right)$ are on the plate nodes, which means $V$ has nontrivial solution, the determinant of $C$ must be set zero. $\operatorname{det}(C)=0$ is the characteristic equation to determine the eigenfrequency $\omega$ of the plate carrying concentrated masses and springs. Equation (45) by FSTM formulation in essence is an eigenvalue problem of an $(r+s) \times(r+s)$ matrix. It also needs to be emphasized that the FSTM can only be applied to the plate with the four edges simply supported. Because the double sine series expansion of the plate transverse deflection in Eq. (38) is only applicable to the plate with the four edges simply supported, Eq. (42) cannot be obtained without this double sine series expansion.

3.2 Computation Example. Figure 4 is a schematic diagram 


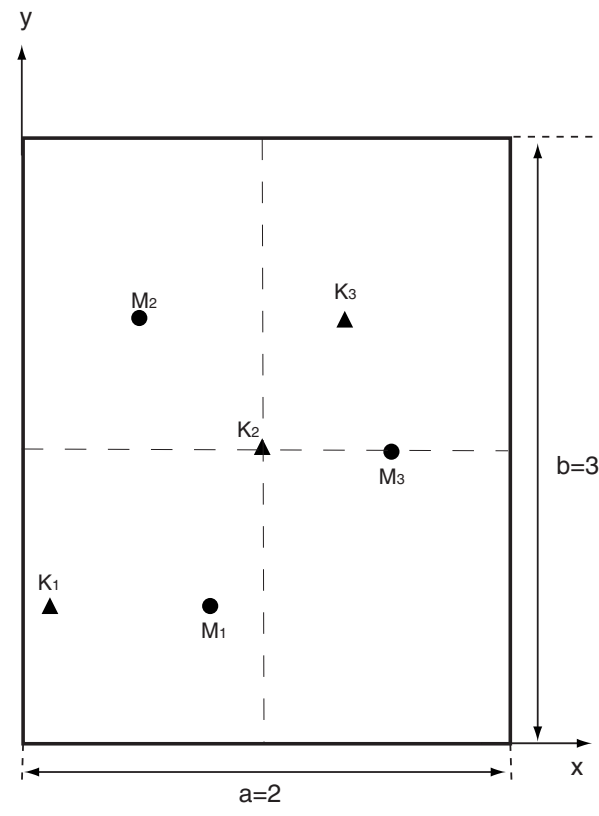

Fig. 4 The schematic diagram of the distribution of the concentrated masses and springs in the plate

of the rectangular plate carrying three concentrated masses and three concentrated springs. All the following parameters related with the plate and concentrated mass/spring are taken from $\mathrm{Wu}$ and Luo's paper [30] in order to have a comparison with their ANCM and FEM results.

$$
\begin{gathered}
a=2.0 \mathrm{~m}, \quad b=3.0 \mathrm{~m}, \quad h=0.005 \mathrm{~m} \\
E=2.051 \times 10^{11} \mathrm{~N} / \mathrm{m}^{2}, \quad M=39.25 \mathrm{~kg} / \mathrm{m}^{2}, \quad \nu=0.3 \\
M_{1}=70.0 \mathrm{~kg}, \quad u_{1}=0.375 a, \quad v_{1}=0.25 b, \quad M_{2}=50.0 \mathrm{~kg} \\
u_{2}=0.5 a, \quad v_{2}=0.625 b \\
M_{3}=60.0 \mathrm{~kg}, \quad u_{3}=0.75 a, \quad v_{3}=0.5 b, \quad K_{1}=10^{6} \mathrm{~N} / \mathrm{m} \\
a_{1}=0.125 a, \quad b_{1}=0.25 b \\
K_{2}=10^{4} \mathrm{~N} / \mathrm{m}, \quad a_{2}=0.5 a, \quad b_{2}=0.5 b, \quad K_{3}=10^{5} \mathrm{~N} / \mathrm{m} \\
a_{3}=0.625 a, \quad b_{3}=0.625 b
\end{gathered}
$$

Although $\psi(x, y)$ in Eq. (38) is symbolically expressed by the mode summation from $m=1$ to $\infty$ and $n=1$ to $\infty$, only finite number of mode can be used during the computation. Generally, the modes with the lowest eigenfrequencies should be given the priority to be used in the modal expansion of $\psi(x, y)$. For a uniform plate with four edges simply supported, the eigenfrequency $\kappa_{m n}$ is given as follows [42]:

$$
a^{2} \sqrt{\frac{M}{D}} \kappa_{m n}=\pi^{2}\left[m^{2}+n^{2}\left(\frac{a}{b}\right)^{2}\right]
$$

Equation (48) provides the idea on how to take the modes with the lowest frequencies. With this formula and plate dimensions given above, the first nine modes with the lowest eigenfrequencies are $1-1,1-2,2-1,1-3,2-2,2-3,1-4,3-1$, and 3-2 modes $(m-n$ mode in this case is $\sin (m \pi x / a) \sin (n \pi y / b))$. However, in order to compare with $\mathrm{Wu}$ and Luo's ANCM results [30], $m$ is simply taken from 1 to 6 and $n$ is taken from 1 to 5 . So there are a total of 30 modes used in computation. The reason of taking so many modes in computation is that the concentrated mass/spring can severely distort the mode shapes of a uniform plate and large mode number is required to have a good approximation for the real dynamic deflection curve.

Newton-Rhapson method [37] is also used to solve the characteristic equation of $\operatorname{det}(C)=0$ to find the eigenfrequencies. The first six lowest eigenfrequencies obtained by FSTM are given in Table 3 to be compared with Wu and Luo's results by FEM and ANCM [30].

The first four lowest eigenfrequencies obtained by FSTM agree well with Wu and Luo's by both FEM and ANCM. But $\omega_{5}$ differs significantly. It is noticed that $\omega_{6}$ of FSTM is very close to $\mathrm{Wu}$ and Luo's $\omega_{5}$. When Newton-Rhapson method is used to solve the eigenvalue problem of $\operatorname{det}(C)=0$, the upper and lower bounds of each eigenfrequency must be carefully chosen before starting the computation [37]. Otherwise, Newton-Rhapson method may miss the eigenfrequency but find another.

Wu and Luo's FEM and ANCM formulations are an eigenvalue problem of a $175 \times 175$ matrix (175 elements are used in the FEM formulation) and a $30 \times 30$ matrix (30 modes are used in the ANCM formulation) [30], respectively. Although 30 modes are also used in FSTM, the computation by the FSTM formulation mainly depends on the total number of concentrated mass and spring as reflected in Eq. (45). So here the FSTM in essence is an eigenvalue problem of a $6 \times 6$ matrix.

\section{Concluding Remarks}

The analytical method is capable of computing the eigenfrequencies of the beam carrying arbitrary concentrated mass/spring at arbitrary location with high accuracy. Its eigenfrequency computation is to solve an eigenvalue problem of $4(r+s+1) \times 4(r+s$ $+1)$ matrix $(r+s$ is the total number of concentrated mass and spring). However, its eigenvalue problem formulation is relatively difficult, which in general needs to be derived case by case. The Galerkin method, FMTM, and FSTM use the mode shapes of a uniform beam/plate to approximate the deflection curve of the beam/plate carrying concentrated mass/spring. Unlike the Rayleigh method, which requires the construction/guess of a close approximation deflection curve to guarantee the accuracy, the Galerkin method, FMTM, and FSTM can systematically obtain the better approximation deflection curve by incorporating more modes. Compared with the analytical method, the Galerkin method, FMTM, and FSTM can all easily and systematically formulate the eigenvalue problem. Because of the different formulations, the Galerkin method is an eigenvalue problem of an $N$

$\times N$ matrix ( $N$ is the mode number), and FMTM and FSTM are eigenvalue problems of an $(r+s) \times(r+s)$ matrix. As demonstrated

Table 3 Comparison of the plate eigenfrequencies (rad/s) computed by FEM, ANCM and FSTM

\begin{tabular}{lcccccc}
\hline \hline Methods/eigenfrequency & $\omega_{1}$ & $\omega_{2}$ & $\omega_{3}$ & $\omega_{4}$ & $\omega_{5}$ & $\omega_{6}$ \\
\hline FEM (Ref. [30]) & 28.831 & 39.775 & 47.158 & 82.898 & 105.352 & Not available \\
ANCM (Ref. [30]) & 28.632 & 39.392 & 48.084 & 81.638 & 104.038 & Not available \\
FSTM (this paper) & 27.564 & 39.767 & 50.071 & 84.813 & 95.414 & 103.164 \\
\hline \hline
\end{tabular}


in the computation example, FMTM shows good convergence and accuracy when the concentrated mass is large. With large concentrated mass or large spring stiffness, which severely distorts the mode shape of a uniform beam/plate and needs large number of mode shapes to approximate the deflection, FMTM and FSTM both have the advantages of high accuracy and much less computation. The analytical method has great difficulty of incorporating the damping effect in its eigenfrequency formulation, which is not a problem at all for FMTM, FSTM, and the Galerkin method. But FMTM has the difficulty of computing the case when the concentrated mass is small or close to the node and the clamped/hinged end. Therefore, FMTM is not a good choice for the mass sensor application of detecting tiny accreted mass. In contrast to FMTM, the Galerkin method shows good convergence and accuracy when the concentrated mass is small, but poor convergence and accuracy when the concentrated mass is large. When the concentrated mass is small, the beam deflection shape is not significantly different from the mode shape of a uniform beam; the Galerkin method with one mode approximation (i.e., Eq. (21)) can thus accurately compute the mass sensor eigenfrequency with little computation effort, which makes it very suitable to be used in the mass sensor application of detecting tiny accreted mass.

\section{Acknowledgment}

This work is supported by the National Natural Science Foundation of China (NSFC) (Grant No. 10721202) and the LNM initial funding for young investigators.

\section{References}

[1] Wenzel, W., and White, R. M., 1989, "Analytical Comparison of the Sensitivities of Bulk-Wave, Surface-Wave, and Flexural Plate-Wave Ultrasonic Gravimetric Sensors," Appl. Phys. Lett., 54, pp. 1976-1978.

[2] Cunningham, B., Weinberg, M., Pepper, J., Clapp, C., Bousquet, R., Hugh, B., Kant, R., Daly, C., and Hauser, E., 2001, "Design, Fabrication and Vapor Characterization of a Micrcofabricated Flexural Plate Resonator Sensor and Application to Integrated Sensor Arrays," Sens. Actuators B, 73, pp. 112-123.

[3] Grate, J. W., Wenzel, S. W., and White, R. M., 1991, "Flexural Plate Wave Devices for Chemical Analysis," Anal. Chem., 63, pp. 1552-1561.

[4] Zellers, E. T., Batterman, S. A., Han, M., and Patrash, S. J., 1995, "Optimal Coating Selection for the Analysis of Organic Vapor Mixtures With PolymerCoated Surface Acoustic Wave Sensor Arrays," Anal. Chem., 67, pp. 1092 1106.

[5] Ramos, D., Tamayo, J., Mertens, J., Calleja, M., and Zaballos, A., 2006, "Origin of the Response of Nanomechanical Resonators to Bacteria Adsorption," J. Appl. Phys., 100, pp. 106105.

[6] Ilic, B., Yang, Y., and Craighead, H. G., 2004, "Virus Detection Using Nanoelectromechanical Devices,” Appl. Phys. Lett., 85, pp. 2604-2606.

[7] Verbridge, S. S., Parpia, J. M., Reichenbach, R. B., Bellan, L. M., and Craighead, H. G., 2006, "High Quality Factor Resonance at Room Temperature With Nanostrings Under High Tensile Stress," J. Appl. Phys., 99, p. 124304.

[8] Ekinci, K. L., and Roukes, M. L., 2005, "Nanoelectromechanical Systems," Rev. Sci. Instrum., 76, p. 061101.

[9] Ilic, B., Craighead, H. G., Krylov, S., Senaratne, W., Ober, C., and Neuzil, P., 2004, "Attogram Detection Using Nanoelectromechanical Oscillators," J. Appl. Phys., 95, pp. 3694-3703.

[10] Wu, D. H., Chien, W. T., Chen, C. S., and Chen, H. H., 2006, "Resonant Frequency Analysis of Fixed-Free Single-Walled Carbon Nanotube-Based Mass Sensor," Sens. Actuators, A, 126, pp. 117-121.

[11] Dohn, S., Sandberg, R., Svendsen, W., and Boisen, A., 2005, "Enhanced Functionality of Cantilever Based Mass Sensors Using Higher Modes," Appl. Phys. Lett., 86, p. 233501.

[12] Jin, D., Li, X., Liu, J., Zuo, G., Wang, Y., Liu, M., and Yu, H., 2006, "HighMode Resonant Piezoresistive Cantilever Sensors for Tens-Femtogram Resoluble Mass Sensing in Air," J. Micromech. Microeng., 16, pp. 1017-1023.

[13] Ono, T., and Esashi, M., 2005, "Stress-Induced Mass Detection With a
Micromechanical/Nanomechanical Silicon Resonator,” Rev. Sci. Instrum., 76, pp. 093107.

[14] Ekinci, K. L., Huang, X. M. H., and Roukes, M. L., 2004, "Ultrasensitive Nanoelectromechanical Mass Detection,” Appl. Phys. Lett., 84, pp. 44694471 .

[15] Ekinci, K. L., Yang, Y. T., and Roukes, M. L., 2004, "Ultimate Limits to Inertial Mass Sensing Based Upon Nanoelectromechanical Systems," J. Appl. Phys., 95, pp. 2682-2689.

[16] Tseytlin, Y. M., 2005, "High Resonant Mass Sensor Evaluation: An Effective Method," Rev. Sci. Instrum., 76, p. 115101.

[17] Lavrik, N. V., Sepaniak, M. J., and Datskos, P. G., 2004, "Cantilever Transducers as a Platform for Chemical and Biological Sensors," Rev. Sci. Instrum., 75, pp. 2229-2253.

[18] Zhang, Y., and Zhao, Y., 2006, "Numerical and Analytical Study on the Pull-In Instability of Micro-Structure Under Electrostatic Loading," Sens. Actuators, A, 127, pp. 366-380.

[19] Timoshenko, S. P., and Young, D. H., 1955, Vibration Problems in Engineering, 3rd ed., D. Van Nostrand Company Inc., New York, Section 1.4.

[20] Low, K. H., 1997, "Closed Form Formulation for Fundamental Vibration Frequency of Beams Under Off-Center Load,” J. Sound Vib., 201, pp. 528-533.

[21] Chen, Y., 1963, "On the Vibration of Beams or Rods Carrying a Concentrated Mass," ASME J. Appl. Mech., 30, pp. 310-311.

[22] Low, K. H., 1998, "On the Eigenfrequencies for Mass Loaded Beams Under Classical Boundary Conditions," J. Sound Vib., 215, pp. 381-389.

[23] Srinath, L. S., and Das, Y. C., 1967, "Vibrations of Beams Carrying Mass," ASME J. Appl. Mech., 34, pp. 784-785.

[24] Baker, W. E., 1964, "Vibration Frequencies for Uniform Beams With Central Masses," ASME J. Appl. Mech., 31, pp. 335-337.

[25] Hess, M. S., 1964, "Vibrations Frequencies for a Uniform Beam With Central Mass and Elastic Supports," ASME J. Appl. Mech., 31, pp. 556-558.

[26] Plaut, R. H., Murphy, K. D., and Virgin, L. N., 1995, "Curve and Surface Veering for a Braced Column," J. Sound Vib., 187, pp. 879-885.

[27] Murphy, K. D., and Zhang, Y., 2000, "Vibration and Stability of a Cracked Translating Beam," J. Sound Vib., 237, pp. 319-335.

[28] Wickert, J. A., and Mote, C. D., Jr., 1988, "Linear Transverse Vibration of Axially Moving String-Particle System,” J. Acoust. Soc. Am., 84, pp. 963969.

[29] Amba-Rao, C. L., 1964, "On the Vibration of a Rectangular Plate Carrying a Concentrated Mass," ASME J. Appl. Mech., 31, pp. 550-551.

[30] Wu, J. S., and Luo, S. S., 1997, "Use of the Analytical-and-Numerical Combined Method in the Free Vibration Analysis of a Rectangular Plate With Any Number of Point Masses and Translational Springs," J. Sound Vib., 200, pp. 179-194.

[31] Chiba, M., and Sugimoto, T., 2003, "Vibration Characteristics of a Cantilever Plate With Attached Spring-Mass System,” J. Sound Vib., 260, pp. 237-263.

[32] Magrab, E. B., 1968, "Vibration of a Rectangular Plate Carrying a Concentrated Mass," ASME J. Appl. Mech., 35, pp. 411-412.

[33] Bergman, L. A., Hall, J. K., Lueschen, G. G. G., and McFarland, D. M., 1993, "Dynamic Green's Function for Levy Plates," J. Sound Vib., 162, pp. 281310 .

[34] Li, Q. S., Li, G. Q., and Liu, D. K., 2000, "Exact Solutions for Longitudinal Vibration of Rods Coupled by Translational Springs," Int. J. Mech. Sci., 42, pp. $1135-1152$.

[35] Li, Q. S., 2003, “An Exact Approach for Free Vibration Analysis of Rectangular Plates With Line-Concentrated Mass and Elastic Line-Support," Int. J. Mech. Sci., 45, pp. 669-685.

[36] Chang, T. C., and Craig, R. R., Jr., 1969, "Normal Modes of Uniform Beams," J. Eng. Mech., 195, pp. 1027-1031.

[37] Press, W. H., Flannery, B. P., Teukolsky, S. A., and Vettering, W. T., 1986, Numerical Recipes, Cambridge University Press, Cambridge, UK.

[38] Senba, A., and Furuya, H., 2008, "Self-Identification Experiments Using Variable Inertia Systems for Flexible Beam Structures," ASME J. Vibr. Acoust., 130, p. 011006.

[39] Cha, P. D., Chan, M., and Nielsen, G., 2008, "Eigenfrequencies of an Arbitrarily Supported Beam Carrying Multiple In-Span Elastic Rod-Mass Systems," ASME J. Vibr. Acoust., 130, pp. 061008.

[40] Sandberg, R., Svendsen, W., Mølhave, K., and Boisen, A., 2005, "Temperature and Pressure Dependence of Resonance in Multi-Layer Microcantilevers," J. Micromech. Microeng., 15, pp. 1454-1458.

[41] Meirovitch, L., 1980, Computational Methods in Structural Dynamics, Sijthoff \& Noordhoff Inc., Rockville, MD.

[42] Gorman, D. J., 1982, Free Vibration Analysis of Rectangular Plates, Elsevier, New York. 